\title{
Clinical features of internal hernia after gastrectomy for gastric cancer
}

\author{
Hong-min Ahn $n^{1,2}$, Si-Hak Lee ${ }^{1,2,3}$, Tae Yong Jeon ${ }^{3,4}$, Dae Hwan Kim³,4, Chang In Choi ${ }^{3,4}$, Su Jin Kim, ${ }^{3,5}$, Cheol Woong Choij, \\ Tae Un Kim, ${ }^{3,6}$ Ki Hyun Kim7, Sun-Hwi Hwang ${ }^{1,2,3}$ \\ ${ }^{1}$ Department of Surgery, Pusan National University Yangsan Hospital, Yangsan, Korea \\ ${ }^{2}$ Research Institute for Convergence of Biomedical Science and Technology, Pusan National University Yangsan Hospital, Yangsan, Korea \\ ${ }^{3}$ Department of Surgery, Pusan National University School of Medicine, Yangsan, Korea \\ ${ }^{4}$ Department of Surgery, Pusan National University Hospital, Busan, Korea \\ ${ }^{5}$ Department of Internal Medicine, Pusan National University Yangsan Hospital, Yangsan, Korea \\ ${ }^{6}$ Department of Radiology, Pusan National University Yangsan Hospital, Yangsan, Korea \\ 'Department of Surgery, Kosin University Gospel Hospital, Busan, Korea
}

Purpose: Internal hernia after gastrectomy is a rare but potentially life-threatening condition without surgical intervention. Clinical risk factors of internal hernia should, hence, be reviewed after gastrectomy. From 2008 to 2018, patients who underwent gastrectomy for gastric cancer were investigated.

Methods: Abdominal computed tomography (CT) was used to screen for internal hernia, and surgical exploration was performed to confirm the diagnosis. Using retrospective statistical analysis, the incidence, characteristics, and risk factors were identified, and the characteristics of the internal hernia group were reviewed.

Results: The overall incidence of internal hernia was $0.9 \%$. From statistical analysis, it was found that laparoscopic surgery was almost five times riskier than open gastrectomy (odds ratio [OR], 4.947; 95\% confidence interval [CI], 1.308-18.710; $p=0.019)$. Body mass index $<25 \mathrm{~kg} / \mathrm{m}^{2}(\mathrm{OR}, 4.596$; 95\% CI, 1.056 20.004; $p=0.042$ ) and proximal gastrectomy (OR, 4.238; 95\% CI, 1.072-16.751; $p=0.039$ ) were also associated with internal hernia. Among 20 patients with internal hernia, 12 underwent laparotomy, and five had their bowels removed due to ischemia. All patients with bowel resected had suffered from short bowel syndrome.

Conclusion: Suspecting an internal hernia should be an important step when a patient with a history of laparoscopic gastrectomy visits for medical care. When suspected, emergent screening through CT scan and surgical intervention should be considered as soon as possible to prevent lifetime complications accordingly.
Received August 31, 2020

Revised 1st October 24, 2020 2nd November 5, 2020 3rd November 20, 2020 4th November 24, 2020

Accepted November 24, 2020

\section{Corresponding author}

Sun-Hwi Hwang

Department of Surgery, Pusan

National University School of

Medicine, 20 Geumo-ro, Mulgeum-

eup, Yangsan 50612, Korea

Tel: +82-55-360-2124

Fax: $+82-55-360-2154$

E-mail: hwangsh@pusan.ac.kr ORCID:

https://orcid.org/0000-0001-8058-0524

Keywords: Abdominal hernia, Gastrectomy, Laparoscopy, Stomach neoplasms, Short bowel syndrome

This is an Open Access article distributed under the terms of the Creative Commons Attribution Non-Commercial License (http:// creativecommons.org/licenses/by-nc/4.0/) which permits unrestricted non-commercial use, distribution, and reproduction in any Copyright ( 2021 The Journal of Minimally medium, provided the original work is properly cited.

\section{INTRODUCTION}

Internal hernia after gastrectomy is defined as herniation of the small bowel through mesenteric defects that can be produced by bowel reconstruction after gastric surgery [1]. Internal hernia may lead to closed-loop obstruction that can be life-threatening 
without surgical intervention. There could be various types of mesenteric defects based on the approach that a surgeon may choose for reconstruction after gastrectomy. The antecolic approach creates two mesenteric defects: Petersen's space, between the Roux limb and the transverse colon, and a defect around the jejunojejunostomy. In Billroth II anastomosis, Petersen's space is referred to as the defect between the jejunal loop and the transverse colon. Another mesenteric defect can be created by retrocolic approach anastomosis; the defect is within the transverse mesocolon [1,2]. During or after recovery, bowel movement causes small intestines to herniate into one of these defects, eventually giving rise to an internal hernia.

Laparoscopic surgery has become the treatment of choice for early gastric cancer rather than open abdominal surgery. Unfortunately, postoperative intraabdominal adhesion formation after laparoscopic surgery is markedly less than that after open abdominal surgery. The patterns of adhesions are also different based on the surgical approach. After laparoscopic surgery, adhesions form between the abdominal wall and the bowels, as opposed to visceral adhesions, which commonly occur after open abdominal surgery [3]. Less adhesion formation explains the higher motility of bowel, which creates the possibility of herniation through mesenteric defects. Following the concept of adhesion formation, iatrogenic adhesions within the mesenteric defects have been attempted to prevent internal hernias. Many promising results have shown that closure of mesenteric defects with either absorbable or non-absorbable sutures helps prevent internal hernia [4]. However, this late complication still occurs and threatens patients' lives.

In the surgical treatment of morbid obesity, internal hernia is a common complication after laparoscopic procedures $[5,6]$. Nevertheless, in this study, we focused on gastrectomy for gastric cancer, and the characteristics and clinical factors of internal hernia after gastric cancer surgery. Based on this review of internal hernia cases, we would like to suggest steps for management of internal hernia after gastric cancer surgery.

\section{METHODS}

\section{Patients}

From December 2008 to December 2018, the electronic charts of 2,599 patients were retrospectively reviewed. The inclusion criteria were age of 19 to 80 years, diagnosis of gastric cancer, having undergone gastrectomy based on the location of the cancer. A total of 319 patients who underwent Billroth I reconstruction were excluded because no mesenteric defects could be generated after the anastomosis. We also excluded 10 other patients whose surgery was discontinued due to the confirmation of carcinomatosis peritonei during surgical exploration. Eventually, 2,270 patients who underwent gastrectomy with Roux-en-Y (RY) reconstruction, Billroth II reconstruction, or double-tract reconstruction

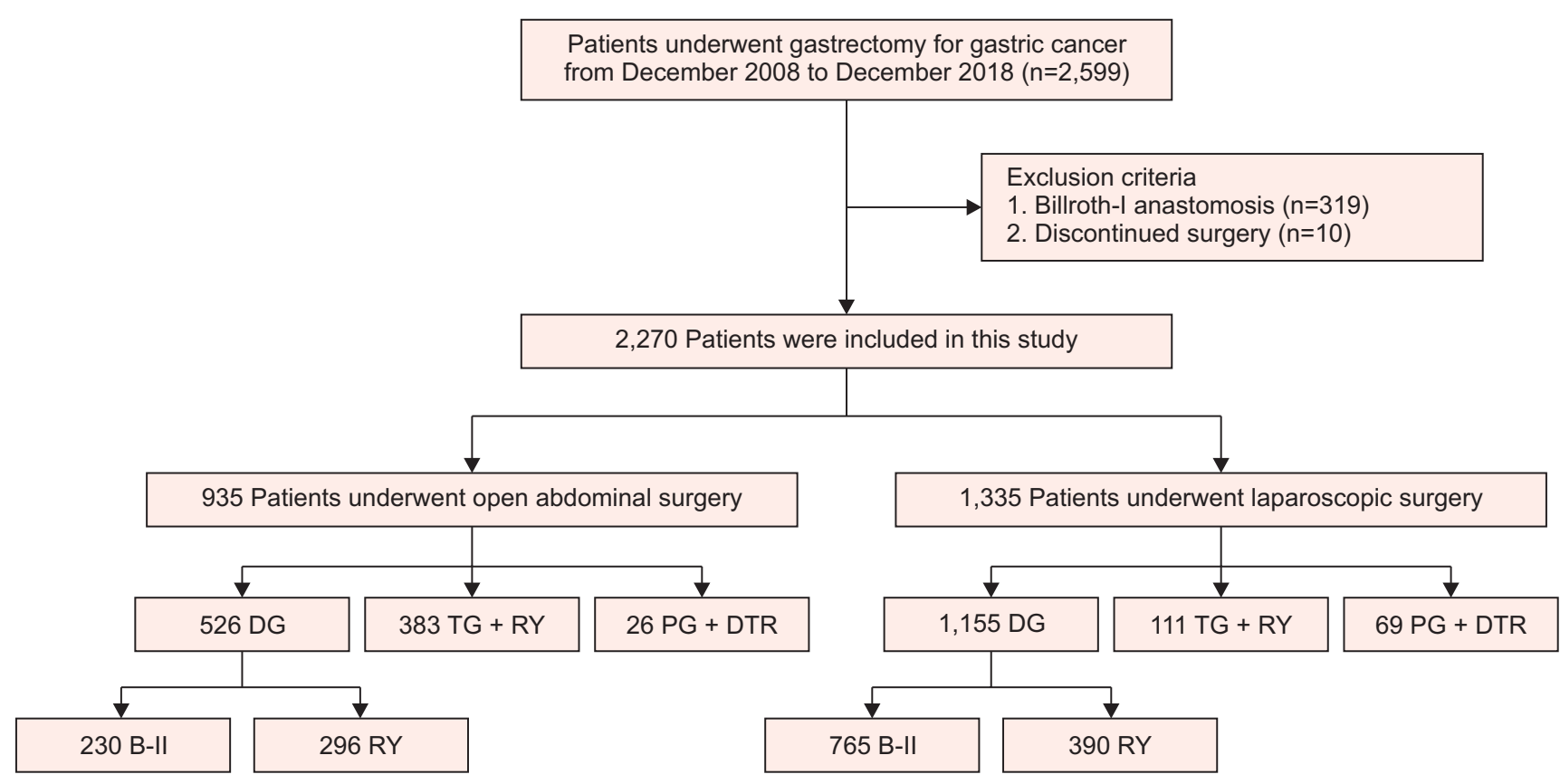

Fig. 1. Patient selection. We reviewed the data of 2,599 patients who underwent gastrectomy for gastric cancer from December of 2008 to 2018 . Three hundred twenty-nine patients were excluded and the remaining 2,270 were investigated for internal hernia. DG, distal gastrectomy; TG, total gastrectomy; RY, Roux-en-Y; PG, proximal gastrectomy; DTR, double-tract reconstruction; B-II, Billroth II. 
(DTR) for gastric cancer were included in the study (Fig. 1). The endpoint of this study was when the patient was diagnosed with internal hernia for the first time after the initial gastric cancer surgery.

\section{Selection of internal hernia}

Abdominal computed tomography (CT) and surgical exploration were performed as diagnostic modalities for internal hernia. The CT results were used to screen for internal hernia after gastrectomy. Following the first operation, all patients underwent abdominal CT for routine postoperative regular check-up for cancer recurrence. During or after admission for initial surgery, when a patient showed abnormal symptoms such as severe abdominal pain, fever, nausea, or vomiting, associated with abnormal follow-up laboratory studies including elevated white blood cell (WBC) counts or C-reactive protein (CRP) levels, the patient underwent abdominal CT for further evaluation. When the imaging study showed a whirl sign, Petersen's hernia or internal hernia, the patient was suspected as having internal hernia. The final diagnosis was confirmed by surgical exploration, either open or laparoscopic surgery.

There are many CT signs that suggest internal hernia, such as the mesenteric whirl sign (or swirl sign), the mushroom sign, hurricane eye, small bowel obstruction, clustered loops, bowel behind the superior mesenteric artery, right-sided anastomosis, engorged nodes, superior mesenteric vein beaking, criss-cross, and others [7-10]. Previous radiologic studies suggest that the whirl sign is the most predictive sign, with high interobserver agreement, regardless of the level of training [8]. Thus, we chose the mesenteric whirl sign on abdominal CT as an indication for internal hernia, even in the context of normal laboratory studies and physical examinations (Fig. 2).

\section{Management of internal hernia}

We reviewed patients' charts to determine the time interval for internal hernia occurrence from initial surgery to screening for internal hernia by CT scan. Only with the surgical intervention, we confirmed the internal hernia and we chose either laparoscopic exploration or explorative laparotomy as the surgical intervention depending on the degree of bowel distension and the presence or absence of bowel wall enhancement from the CT scan. The treatment of internal hernia was determined by exploring the conditions of the herniated bowel. Bowel resection and anastomosis were performed with necrotic herniated bowels. When bowel conditions were intact, however, manual reduction and closure of mesenteric defects were performed with consideration of a second-look laparotomy within 24 to 48 hours.

\section{Statistical analysis}

Descriptive statistics including frequency were used to identify the incidence of internal hernia. Chi-square and Fisher exact tests were used to identify the risk factors of internal hernia. Sex, current smoking state, body mass index (BMI) at the time of initial surgery, types of gastrectomy, surgical approach for gastrectomy, pathologic stage of cancer, and American Society of Anesthesiologists (ASA) physical status classification were considered as variables for analysis. For multivariate analysis, logistic regression analysis was used accordingly. The covariance input criterion was less than 0.1 , and the elimination criterion was less than 0.05 . For subgroup analysis of patients with internal hernia, the chi-square test, Fisher exact test, and Mann-Whitney $U$ test were performed. IBM SPSS version 23.0 for Windows (IBM Corp., Armonk, NY, USA) was used to analyze the data. All results with a $p$ value of less than 0.05 were considered significant.
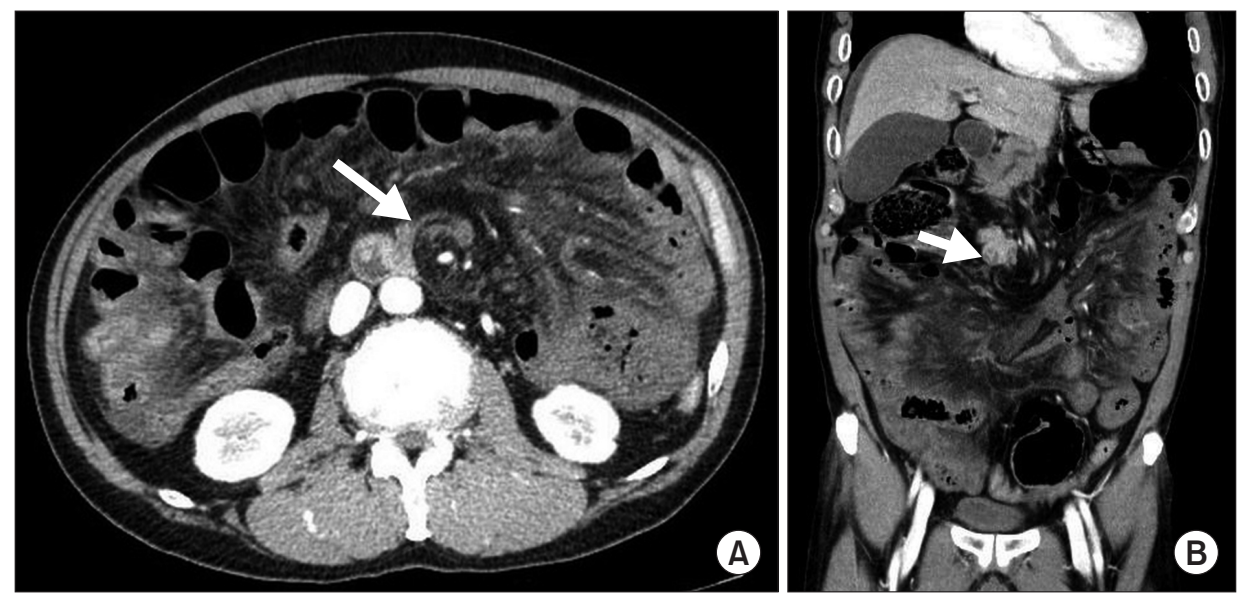

Fig. 2. The whirl sign from the abdominal computed tomography. Among many radiologic signs, the mesenteric whir sign is known as the most predictive sign for screening the internal hernia after gastric surgery. White arrows indicate mesenteric whirl signs from axial view $(A)$ and coronal view (B). 


\section{RESULTS}

We analyzed data from 2,270 patients who underwent gastrectomy for gastric cancer, and the basic characteristics are shown in Table 1. Among them, 935 patients (41.2\%) underwent open gastrectomy surgery and 1,335 (58.8\%) underwent laparoscopic

Table 1. Characteristics of patients received gastrectomy

\begin{tabular}{|c|c|}
\hline Variable & Value \\
\hline No. of patients & 2,270 \\
\hline \multicolumn{2}{|l|}{ Sex } \\
\hline Male & $1,497(65.9)$ \\
\hline Female & 773 (34.1) \\
\hline Age (yr) & $61.2 \pm 11.1$ \\
\hline \multicolumn{2}{|l|}{ Body mass index $\left(\mathrm{kg} / \mathrm{m}^{2}\right)$} \\
\hline$\geq 25$ & 758 (33.4) \\
\hline$<25$ & $1,512(66.6)$ \\
\hline \multicolumn{2}{|l|}{ Current smoking status } \\
\hline No & $1,790(78.9)$ \\
\hline Yes & $480(21.1)$ \\
\hline \multicolumn{2}{|l|}{ Type of gastrectomy } \\
\hline Distal gastrectomy & $1,681(74.1)$ \\
\hline Total gastrectomy & 494 (21.8) \\
\hline Proximal gastrectomy & $95(4.2)$ \\
\hline \multicolumn{2}{|l|}{ Surgical approach } \\
\hline Open & $935(41.2)$ \\
\hline Laparoscopy & $1,335(58.8)$ \\
\hline \multicolumn{2}{|l|}{ Pathologic cancer stage } \\
\hline 1 & $1,472(64.8)$ \\
\hline$\|$ & $336(14.8)$ \\
\hline III & $448(19.7)$ \\
\hline IV & $14(0.6)$ \\
\hline \multicolumn{2}{|l|}{ ASA PS classification } \\
\hline I & $838(36.9)$ \\
\hline$\|$ & $1,329(58.5)$ \\
\hline$\|$ & $100(4.4)$ \\
\hline IV & $3(0.1)$ \\
\hline \multicolumn{2}{|l|}{ Internal hernia } \\
\hline No & $2,250(99.1)$ \\
\hline Yes & $20(0.9)$ \\
\hline
\end{tabular}

Values are presented as number only, number $(\%)$, or mean \pm standard deviation.

ASA PS, American Society of Anesthesiologists physical status. gastrectomy. Distal gastrectomy was performed in 1,681 patients (74.1\%), 494 patients underwent total gastrectomy, and 95 patients underwent proximal gastrectomy with DTR for gastric cancer. Internal hernia was found in 20 patients, with an overall incidence of $0.9 \%$. These 20 patients were investigated separately, and their clinical characteristics are shown in Table 2 . The mean time interval between initial gastrectomy and internal hernia occurrence was 28 months (standard deviation, 25.6 months). All 20 patients underwent surgical intervention. Eight patients were treated only with laparoscopic reduction of the internal hernia with mesenteric defect repair. The other 12 patients underwent explorative laparotomy to treat the internal hernia; among them, five underwent bowel resection because of bowel ischemia and necrosis.

Based on the results of univariate analysis, initial BMI, types of gastrectomy, and surgical approach methods were individually associated with the occurrence of internal hernia. In particular with the surgical approach of gastrectomy, only $0.3 \%$ experienced internal hernia in the open gastrectomy group, while $1.3 \%$ experienced internal hernia in the laparoscopic gastrectomy group ( $p$ $=0.017$ ). On multivariate analysis, the difference in incidence of internal hernia between open and laparoscopic gastrectomy was statistically significant (odds ratio [OR], 4.947; 95\% confidence interval [CI], 1.308-18.710; $p=0.019$ ). In addition to the approach methods of gastrectomy, BMI, and types of gastrectomy also played a role in cases of internal hernia. Patients with a BMI below $25 \mathrm{~kg} / \mathrm{m}^{2}$ had significantly more internal hernias than those

Table 2. Characteristics of internal hernia after gastrectomy for gastric cancer

\begin{tabular}{lr}
\multicolumn{1}{c}{ Characteristic } & \multicolumn{1}{c}{ Value } \\
\hline Age $(y r)$ & $64.0 \pm 11.2$ \\
Sex & $13(65.0)$ \\
Male & $7(35.0)$ \\
Female & $28.0 \pm 25.6$ \\
Interval from initial surgery to internal hernia (mo) & $8(40.0)$ \\
Treatment of internal hernia & $12(60.0)$ \\
Laparoscopic reduction & \\
Explorative laparotomy & $18(90.0)$ \\
Internal hernia site & $2(10.0)$ \\
Petersen & \\
Jejunojejunostomy & $5(25.0)$ \\
Bowel resection & $15(75.0)$ \\
\hline Yes & \\
No & \\
\hline
\end{tabular}

Values are presented as mean \pm standard deviation or number (\%). 
Table 3. Univariate and multivariate analysis for associated factors of internal hernia

\begin{tabular}{|c|c|c|c|c|c|c|}
\hline \multirow{2}{*}{ Variable } & \multirow{2}{*}{$\begin{array}{c}\text { Without } \\
\text { internal hernia }\end{array}$} & \multirow{2}{*}{$\begin{array}{c}\text { With } \\
\text { Internal hernia }\end{array}$} & \multirow{2}{*}{$p$ value } & \multicolumn{3}{|c|}{ Multivariate analysis } \\
\hline & & & & $\mathrm{OR}$ & $95 \% \mathrm{Cl}$ & $p$ value \\
\hline No. of patients & 2,250 & 20 & & & & \\
\hline Sex & & & 0.928 & & & \\
\hline Male & $1,484(99.1)$ & $13(0.9)$ & & & & \\
\hline Female & 766 (99.1) & $7(0.9)$ & & & & \\
\hline Current smoking & & & 1.000 & & & \\
\hline No & $1,774(99.1)$ & $16(0.9)$ & & & & \\
\hline Yes & 476 (99.2) & $4(0.8)$ & & & & \\
\hline Initial BMI $\left(\mathrm{kg} / \mathrm{m}^{2}\right)$ & & & $0.026^{*}$ & & & \\
\hline$\geq 25$ & 756 (99.7) & $2(0.3)$ & & 1.000 & & \\
\hline$<25$ & $1,494(98.8)$ & $18(1.2)$ & & 4.596 & 1.056-20.004 & $0.042^{*}$ \\
\hline Types of gastrectomy & & & $<0.001^{*}$ & & & \\
\hline Distal gastrectomy & $1,671(99.4)$ & $10(0.6)$ & & 0.404 & $0.116-1.409$ & 0.155 \\
\hline Total gastrectomy & 490 (99.2) & $4(0.8)$ & & 1.000 & & \\
\hline Proximal gastrectomy & 89 (93.7) & $6(6.3)$ & & 4.238 & $1.072-16.751$ & $0.039^{*}$ \\
\hline Surgical approach & & & $0.017^{\star}$ & & & \\
\hline Open & $932(99.7)$ & $3(0.3)$ & & 1.000 & & \\
\hline Laparoscopy & $1,318(98.7)$ & $17(1.3)$ & & 4.947 & $1.308-18.710$ & $0.019^{\star}$ \\
\hline Pathologic cancer stage & & & 0.401 & & & \\
\hline$H$ & $1,790(99.0)$ & $18(1.0)$ & & & & \\
\hline$\|||-I V$ & 460 (99.6) & $2(0.4)$ & & & & \\
\hline ASA PS classification & & & 1.000 & & & \\
\hline$|-| \mid$ & 2,147 (99.1) & $20(0.9)$ & & & & \\
\hline$\|||-\mid V$ & $103(100)$ & $0(0)$ & & & & \\
\hline
\end{tabular}

Values are presented as number only or number (\%) unless otherwise specified.

$\mathrm{OR}$, odds ratio; $\mathrm{Cl}$, confidence interval; $\mathrm{BMI}$, body mass index; ASA PS, American Society of Anesthesiologists physical status.

*Statistically significant difference $(p<0.05)$.

with a BMI above $25 \mathrm{~kg} / \mathrm{m}^{2}$ (OR, 4.596; 95\% CI, 1.056-20.004; $p=$ 0.042). When we compared the results of total gastrectomy with proximal gastrectomy, we found that patients who underwent proximal gastrectomy had a fourfold higher risk of developing internal hernia (OR, 4.238; 95\% CI, 1.072-16.751; $p=0.039$ ) (Table 3).

Laboratory findings such as WBC counts and CRP levels were compared among the groups. Higher WBC counts were observed in cases of explorative laparotomy than in those of laparoscopic surgery, with statistical significance $(p=0.025)$. There were no statistically significant differences between the bowel resection and manual reduction groups with respect to WBC counts. There were also no correlations between the decision of treatment methods and CRP levels (Fig. 3).

\section{DISCUSSION}

The incidence of internal hernia after gastrectomy for gastric cancer was much lower than the rate after bariatric gastric bypass surgery, which in previous studies was reported to range from $5 \%$ to $9 \%$ [11]. Going by technical differences and various follow-up periods, calculating the definite incidence of internal hernia would be challenging. Nevertheless, integrating the results of recent studies and ours, the incidence of internal hernia after gastric cancer surgery ranges from $0.14 \%$ to $4.2 \%$ [1,12-16]. The Roux limb length difference could be one of the explanations for the lower incidence in gastric cancer surgery than in gastric bypass. Comparing two groups with different Roux limb lengths that underwent gastric bypass surgery, internal hernia occur- 

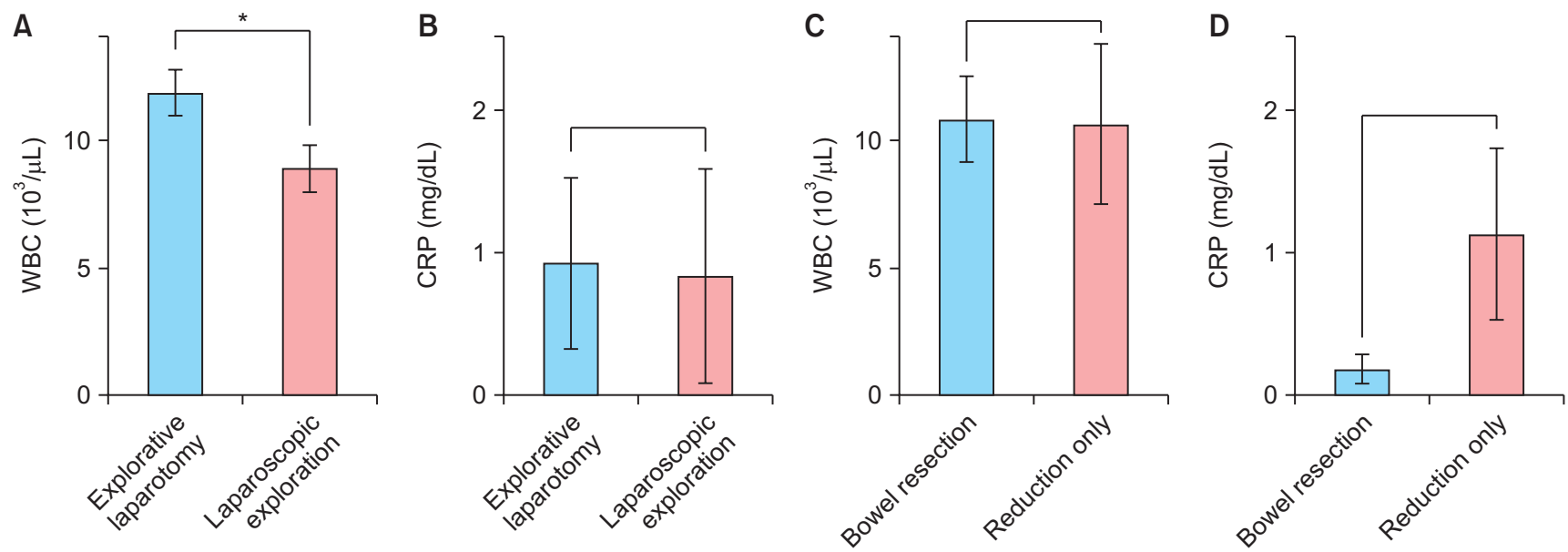

Fig. 3. Laboratory study of internal hernia. White blood cell (WBC) counts and C-reactive protein (CRP) levels were compared between the subgroups to understand the diagnostic modules for choosing the proper management timing and methods. In aggressive treatment methods such as laparotomy and open conversion surgery, WBC counts were significantly higher than that in the laparoscopy group $(p=0.025)(A)$, while CRP levels were irrelevant $(p=0.521)(B)$. (C) Interestingly, even though the statistical significance is irrelevant, WBC counts were similar in both the bowel resection group, due to bowel necrosis, and the reduction only group $(p=0.735)$. (D) CRP levels were even lower in the bowel resection group than in the reduction only group $(p=$ 0.933). ${ }^{*} p<0.05$.

rence was higher in the longer limb group [5,6]. Since shorter Roux limbs are reconstructed in gastric cancer surgery than in gastric bypass surgery, internal hernia could be considered a rare complication after gastrectomy.

Although internal hernia is a rare complication that can occur after gastrectomy, it can be life-threatening because of closedloop obstruction with necrosis of herniated small bowel, which may lead to sepsis and multiorgan failure. Among our internal hernia cases, five patients underwent bowel resection due to severe bowel necrosis. At least $100 \mathrm{~cm}$ of the small bowel had to be removed in these cases. All five patients had short bowel syndrome and needed hospital care for conservative treatment, including total parenteral nutrition. For this reason, it is important not to delay surgical intervention when an internal hernia is suspected.

Based on understanding of the severity of internal hernia, many previous studies have suggested various methods to prevent internal hernia after gastric surgery. Adhesion formation is an important concept to explain internal hernias. Iatrogenic adhesions caused by closure of mesenteric defects have been used to prevent internal hernia. Some studies have tried alternative methods to increase adhesion within mesenteric defects by rubbing the sponge against the mesentery until petechiae appear [17]. Despite the hard work of prevention, internal hernia after gastric surgery still occurs. Partially, technical errors such as loosening the sutured knots can cause re-opening of mesenteric defects; however, the reduction of visceral fat due to excessive weight loss after gastric surgery is a well-known cause of widening the defects [13]. Although closure of mesenteric defects is commonly performed to prevent internal hernia, proper management is still necessary. According to our results, the timing of internal hernia is unpredictable. This is why we suggest the first step of management should be suspicion of internal hernia. In addition to unpredictable features, a low incidence of internal hernia makes it harder to suspect this complication when the patients have severe abdominal pain, nausea, and vomiting with a history of gastrectomy.

After suspecting internal hernia, the next step, in general, would be laboratory and imaging studies. According to our results, laboratory findings are not helpful in determining the surgical approach for managing internal hernia. The most useful screening modality would be abdominal CT with contrast. When a CT scan shows mesenteric whirl sign, the possibility of internal hernia increases. Either with or without the enhancement of bowel wall through the CT scan, it is still important not to hesitate the surgical intervention [18]. Without decreased enhancement, there is possibility of salvaging the herniated bowel. Based on our clinical experience, we have learned that laparoscopic exploration would be a better choice for surgical intervention to explore the inside of the abdomen prior to making a long incision. In most cases of non-necrotic bowel conditions, simple manual reduction and closure of the mesenteric defect would be sufficient for surgical treatment of internal hernia. To standardize the management protocol for internal hernia, however, further study with a control group may be needed.

Clinical factors of internal hernia have been analyzed in many previous studies. Our results show that well-known, clinical factors, such as BMI and laparoscopic surgical technique, also influ- 
ence the incidence of internal hernia. One interesting result from our analysis was the higher incidence of internal hernia after proximal gastrectomy with DTR compared to total gastrectomy with RY anastomosis. Many studies have been conducted comparing proximal gastrectomy with total gastrectomy for proximal gastric cancer; the oncological safety was not significantly different between the proximal and total gastrectomy [19-23]. Focusing on late complications such as internal hernia, the results were different from each other $[19,24]$. Our initial assumption was to form an extra mesenteric defect by performing gastrojejunostomy during DTR. Unfortunately, from our results, $90 \%$ of internal hernia patients were observed with Petersen's defect, and the rest (10\%) were observed with the jejunojejunostomy defect (Table 2). Thus, to establish a comparison of proximal and total gastrectomy by complication occurrence, including internal hernia, further studies are needed with larger numbers of patients.

This study has several limitations, including the varied followup periods for individual patients. Some internal hernia cases might have been lost if the patient visited other facilities at symptom onset. From an academic point of view, this study is retrospective and single-center in nature. Also, it has a small sample size. Despite these limitations, this study was able to achieve statistically valid results by investigating all patients who underwent gastrectomy with RY anastomosis, Billroth II anastomosis, or DTR for gastric cancer. Lastly, from a technical point of view, due to lack of information about closing mesenteric defects and reconstruction approach methods from initial gastrectomy, we were unable to analyze the effects of closing mesenteric defects.

In conclusion, laparoscopy itself is a risk factor for internal hernia after gastric cancer surgery. In addition, patients with a BMI of $<25 \mathrm{~kg} / \mathrm{m}^{2}$ or those who underwent proximal gastrectomy as previous surgery had a higher risk of internal hernia. Thus, it is important to suspect internal hernia when evaluating the cause of new-onset severe abdominal pain, nausea, and vomiting symptoms in patients with a history of laparoscopic gastrectomy. Furthermore, after the suspicion and screening process, emergent surgical intervention would be a practical step to prevent massive bowel resection and life-long complications such as short bowel syndrome.

\section{NOTES}

\section{Ethical statements}

This study was reviewed and approved by Institutional Review Board of Pusan National University Yangsan Hospital (No. 052020-630). Since this study is a retrospective study that reviewed electronic charts and CT readings, and personal information protection measures are well established, need for formal consent from the patients was waived.

\section{Authors' contributions}

Conceptualization: $\mathrm{SHH}$

Data curation: SHH, SHL, KHK, TUK

Formal analysis: SHL, KHK, HA

Funding acquisition: $\mathrm{SHH}, \mathrm{TYJ}, \mathrm{DHK}, \mathrm{CIC}$

Investigation: KHK, HA

Methodology: SHH, SHL, SJK, CWC

Project administration: $\mathrm{SHH}, \mathrm{SHL}$

Visualization: SHH, SHL, HA

Writing-original draft: HA

Writing-review \& editing: SHH, SHL, HA

All authors read and approved the final manuscript.

\section{Conflict of interest}

All authors have no conflicts of interest to declare.

\section{ORCID}

Hong-min Ahn, https://orcid.org/0000-0001-9963-2021

Si-Hak Lee, https://orcid.org/0000-0002-1923-4777

Tae Yong Jeon, https://orcid.org/0000-0002-4481-7982

Dae Hwan Kim, https://orcid.org/0000-0001-7450-797X

Chang In Choi, https://orcid.org/0000-0002-1920-1879

Su Jin Kim, https://orcid.org/0000-0003-3816-9664

Cheol Woong Choi, https://orcid.org/0000-0001-8867-3039

Tae Un Kim, https://orcid.org/0000-0003-1017-6926

Ki Hyun Kim, https://orcid.org/0000-0002-8296-3307

Sun-Hwi Hwang, https://orcid.org/0000-0001-8058-0524

\section{REFERENCES}

1. Han WH, Eom BW, Yoon HM, Kim YW, Ryu KW. Clinical characteristics and surgical outcomes of internal hernia after gastrectomy in gastric cancer patients: retrospective case control study. Surg Endosc 2019;33:2873-2879.

2. Steele KE, Prokopowicz GP, Magnuson T, Lidor A, Schweitzer M. Laparoscopic antecolic Roux-en-Y gastric bypass with closure of internal defects leads to fewer internal hernias than the retrocolic approach. Surg Endosc 2008;22:2056-2061.

3. Schäfer M, Krähenbhl L, Büchler MW. Comparison of adhesion formation in open and laparoscopic surgery. Dig Surg 1998;15:148-152.

4. Stenberg E, Szabo E, Ågren G, et al. Closure of mesenteric defects in laparoscopic gastric bypass: a multicentre, randomised, parallel, openlabel trial. Lancet 2016;387:1397-1404.

5. Inabnet WB, Quinn T, Gagner M, Urban M, Pomp A. Laparoscopic Roux-en-Y gastric bypass in patients with $\mathrm{BMI}<50$ : a prospective randomized trial comparing short and long limb lengths. Obes Surg 2005;15:51-57. 
6. Dogan K, Homan J, Aarts EO, van Laarhoven CJ, Janssen IM, Berends FJ. A short or a long Roux limb in gastric bypass surgery: does it matter? Surg Endosc 2017;31:1882-1890.

7. Iannuccilli JD, Grand D, Murphy BL, Evangelista P, Roye GD, MayoSmith W. Sensitivity and specificity of eight CT signs in the preoperative diagnosis of internal mesenteric hernia following Roux-en-Y gastric bypass surgery. Clin Radiol 2009;64:373-380.

8. Kagoma YK, Gayer G. Computed tomography of internal hernias following laparoscopic Roux-en-Y gastric bypass surgery. Semin Ultrasound CT MR 2018;39:145-150.

9. Farukhi MA, Mattingly MS, Clapp B, Tyroch AH. CT scan reliability in detecting internal hernia after gastric bypass. JSLS 2017;21: e2017.00054.

10. Dilauro M, McInnes MD, Schieda N, et al. Internal hernia after laparoscopic Roux-en-Y gastric bypass: optimal CT signs for diagnosis and clinical decision making. Radiology 2017;282:752-760.

11. Kelly KJ, Allen PJ, Brennan MF, Gollub MJ, Coit DG, Strong VE. Internal hernia after gastrectomy for cancer with Roux-Y reconstruction. Surgery 2013;154:305-311.

12. Yoshikawa K, Shimada M, Kurita N, et al. Characteristics of internal hernia after gastrectomy with Roux-en-Y reconstruction for gastric cancer. Surg Endosc 2014;28:1774-1778.

13. Kang KM, Cho YS, Min SH, et al. Internal hernia after gastrectomy for gastric cancer in minimally invasive surgery era. Gastric Cancer 2019;22:1009-1015.

14. Kimura $H$, Ishikawa $M$, Nabae $T$, et al. Internal hernia after laparoscopic gastrectomy with Roux-en-Y reconstruction for gastric cancer. Asian J Surg 2017;40:203-209.

15. Kojima K, Inokuchi M, Kato K, Motoyama K, Sugihara K. Petersen's hernia after laparoscopic distal gastrectomy with Roux-en-Y reconstruction for gastric cancer. Gastric Cancer 2014;17:146-151.
16. Miyagaki $\mathrm{H}$, Takiguchi S, Kurokawa $\mathrm{Y}$, et al. Recent trend of internal hernia occurrence after gastrectomy for gastric cancer. World J Surg 2012;36:851-857.

17. Walker AS, Bingham JR, Causey MW, Sebesta JA. Mesenteric irritation as a means to prevent internal hernia formation after laparoscopic gastric bypass surgery. Am J Surg 2014;207:739-742.

18. Champion JK, Williams M. Small bowel obstruction and internal hernias after laparoscopic Roux-en-Y gastric bypass. Obes Surg 2003; 13:596-600.

19. Jung DH, Lee Y, Kim DW, et al. Laparoscopic proximal gastrectomy with double tract reconstruction is superior to laparoscopic total gastrectomy for proximal early gastric cancer. Surg Endosc 2017;31:39613969.

20. Nozaki I, Hato S, Kobatake T, Ohta K, Kubo Y, Kurita A. Long-term outcome after proximal gastrectomy with jejunal interposition for gastric cancer compared with total gastrectomy. World J Surg 2013; 37:558-564.

21. Masuzawa T, Takiguchi S, Hirao M, et al. Comparison of perioperative and long-term outcomes of total and proximal gastrectomy for early gastric cancer: a multi-institutional retrospective study. World J Surg 2014;38:1100-1106.

22. Ikeguchi M, Kader A, Takaya S, et al. Prognosis of patients with gastric cancer who underwent proximal gastrectomy. Int Surg 2012;97: 275-279.

23. Ahn SH, Lee JH, Park DJ, Kim HH. Comparative study of clinical outcomes between laparoscopy-assisted proximal gastrectomy (LAPG) and laparoscopy-assisted total gastrectomy (LATG) for proximal gastric cancer. Gastric Cancer 2013;16:282-289.

24. Jung DH, Ahn SH, Park DJ, Kim HH. Proximal gastrectomy for gastric cancer. J Gastric Cancer 2015;15:77-86. 